${ }^{1}$ Oles Honchar Dnipro National University

${ }^{2}$ National University, Los Angeles, USA

E-mail: 1kurdachenko@gmail.com, sasha.pypka@gmail.com, isubboti@nu.edu

\title{
On analogs of some classical group-theoretic results in Poisson algebras
}

Presented by Corresponding Member of the NAS of Ukraine V.P. Motornyi

We investigate the Poisson algebras, in which the n-th hypercenter (center) has a finite codimension. It was established that, in this case, the Poisson algebra $P$ includes a finite-dimensional ideal $K$ such that $P / K$ is nilpotent (Abelian). Moreover, if the $n$-th hypercenter of a Poisson algebra $P$ over some field has a finite codimension, and if $P$ does not contain zero divisors, then $P$ is Abelian.

Keyword: Poisson algebra, center, hypercenter, zero divisors, nilpotency.

A Poisson algebra is a vector space $P$ over a field $F$ equipped with two binary operations, the multiplication · and Lie bracket [,] having the following properties:

$$
\begin{aligned}
& a b=b a,(a b) c=a(b c), a(b+c)=a b+a c,(\lambda a) b=a(\lambda b)=\lambda(a b), \\
& {[a+b, c]=[a, c]+[b, c],[a, b+c]=[a, b]+[a, c],[\lambda a, b]=[a, \lambda b]=\lambda[a, b],} \\
& {[a, a]=0,[[a, b], c]+[[b, c], a]+[[c, a], b]=0,} \\
& {[a b, c]=a[b, c]+b[a, c]}
\end{aligned}
$$

for all $a, b, c \in P, \lambda \in F$.

If we will consider $P$ as an associative and commutative algebra by the outer multiplication, addition, and multiplication, then we will denote it by $P(+, \cdot)$. If we will consider $P$ as a Lie algebra by outer multiplication, addition, and Lie bracket, then we will denote it by $P(+,[]$,$) .$

Ци ту в анн я: Kurdachenko L.A., Pypka A.A., Subbotin I.Ya. On analogs of some classical group-theoretic results in Poisson algebras. Допов. Наи. акад. наук Укр. 2021. № 3. С. 11-16. https://doi.org/10.15407/dopovidi 2021.03.011 
Poisson algebras arose from the study of the Poisson geometry [1, 2]. It has appeared in an extremely wide range of areas in mathematics and physics, such as classical and quantum mechanics, quantum groups, quantization theory, Poisson manifolds, algebraic geometry, and operads. Some of the first works, where specific Poisson algebras appeared, were [3-7], while one of the first works, where the study of the properties of abstract Poisson algebras began, was work [8]. Poisson algebras have been and are being studied very intensively by many authors and from various points of view. This paper is devoted to extending some results that became already classical in different algebraic structures to Poisson algebras. The issue that will be discussed here has its sources in articles $[9,10]$. They showed that if the center of a group $G$ has a finite index, then its derived subgroup $[G, G]$ is finite. This result became the starting point for an interesting and broad topic, involving not only groups, but also other algebraic structures, among which were non-associative algebras (Lie algebras and Leibniz algebras) [11]. In particular, it was proved in paper [12] that if the center of a Lie algebra $L$ has finite codimension, then its derived ideal $[L, L]$ has finite dimension. A situation with the center and derived subalgebra in Poisson algebras has significant differences. In the Poisson algebras, the center and derived subalgebra are not ideals. Nevertheless, for Poisson algebras, we obtained a similar result.

We recall some necessary definitions.

Let $P$ be a Poisson algebra over a field $F$. As usual, a subset $B$ of $P$ is called a subalgebra of $P$, if $B$ is a subspace of $P$, and if $x y,[x, y] \in B$ for all $x, y \in B$. A subset $L$ of $P$ is called an ideal of $P$, if $L$ is a subspace of $P$, and if $x b,[x, b] \in L$ for all $b \in L, x \in P$. A Poisson algebra $P$ is called simple, if it has only two ideals $<0>$ and $P$. A Poisson algebra $P$ is called Abelian, if $[x, y]=0$ for all $x, y \in P$.

Let $P$ be a Poisson algebra over a field $F$. Define the lower central series of $P$,

$$
P=\gamma_{1}(P) \geqslant \gamma_{2}(P) \geqslant \ldots \gamma_{\alpha}(P) \geqslant \gamma_{\alpha+1}(P) \geqslant \ldots \gamma_{\delta}(P),
$$

by the following rule: $\gamma_{1}(P)=P, \gamma_{2}(P)=[P, P]$, recursively, $\gamma_{\alpha+1}(P)=\left[\gamma_{\alpha}(P), P\right]$ for all ordinals $\alpha$, and

$$
\gamma_{\lambda}(P)=\bigcap_{\mu<\lambda} \gamma_{\mu}(P)
$$

for all limit ordinals $\lambda$. The last term $\gamma_{\delta}(P)$ is called the lower hypocenter of $P$. We have $\gamma_{\delta}(P)=\left[\gamma_{\delta}(P), P\right]$

We say that a Poisson algebra $P$ is nilpotent, if there exists a positive integer $k$ such that $\gamma_{k}(P)=\langle 0\rangle$. More precisely, $P$ is said to be nilpotent of a nilpotency class $c$, if $\gamma_{c+1}(P)=\langle 0\rangle$, but $\gamma_{c}(P) \neq\langle 0\rangle$.

Put $\zeta(P)=\{z \in P \mid[z, x]=0$ for every $x \in P\}$. The subset $\zeta(P)$ is called the center of $P$. Starting from the center, we can construct the upper central series

$$
\langle 0\rangle=\zeta_{0}(P) \leqslant \zeta_{1}(P) \leqslant \zeta_{2}(P) \leqslant \ldots \zeta_{\alpha}(P) \leqslant \zeta_{\alpha+1}(P) \leqslant \ldots \zeta_{\gamma}(P)=\zeta_{\infty}(P)
$$

of a Poisson algebra $P$ by the following rule: $\zeta_{1}(P)=\zeta(P)$ is the center of $P$, recursively, $\zeta_{\alpha+1}(P) / \zeta_{\alpha}(P)=\zeta\left(P / \zeta_{\alpha}(P)\right)$ for all ordinals $\alpha$, and

$$
\zeta_{\lambda}(P)=\bigcup_{\mu<\lambda} \zeta_{\mu}(P)
$$


for all limit ordinals $\lambda$. We remark that each term of this series is a subalgebra of $P$, which is an ideal of a Lie algebra $P(+,[]$,$) . The last term \zeta_{\infty}(P)$ of this series is called the upper hypercenter of $P$.

We start with some important and useful auxiliary results.

Proposition 1. Let $A$ be an associative commutative algebra over a field $F$ and be generated by a subset $S$. Suppose that a bilinear operation [, ] satisfying conditions $[a, a]=0$ and $[a b, c]=$ $=a[b, c]+b[a, c]$ is defined on $A$. Then $A$ is a Poisson algebra, if and only if $[[a, b], c]+[[b, c], a]$ $+[[c, a], b]=0$ for all $a, b, c \in S$.

Let $A, U$ be Poisson algebras over a field $F$. Then a mapping $f: A \rightarrow U$ is called a homomorphism, if

$$
\begin{aligned}
& f(\lambda a)=\lambda f(a), f(a+b)=f(a)+f(b), \\
& f(a b)=f(a) f(b), f([a, b])=[f(a), f(b)]
\end{aligned}
$$

for all $a, b \in A, \lambda \in F$.

As usual, an injective homomorphism is called a monomorphism, a surjective homomorphism is called an epimorphism, and a bijective homomorphism is called an isomorphism.

Proposition 2. Let $A$ be an arbitrary Poisson algebra over a field F. Then there exists a Poisson algebra $S$ over a field F having a multiplicative identity element and a monomorphism $f: A \rightarrow S$. Moreover, $\operatorname{Im}(f)$ is an ideal of $S$.

This proposition allows us to consider further only Poisson algebras having the multiplicative identity element $1_{P}$.

If $B, C$ are the subspaces of a Poisson algebra $P$, then let

$$
B+C=\{b+c \mid \text { for all } b \in B, c \in C\} ;
$$

$B C$ be the subspace of $\mathrm{P}$ generated by the subset $\{b c \mid$ for all $b \in B, c \in C\}$;

$[B, C]$ be the subspace of $P$, generated by the subset $\{[b, c] \mid$ for all $b \in B, c \in C\}$.

Clearly, $B C$ is a subset of $P$ consisting of elements of the type $a_{1} b_{1}+\ldots+a_{n} b_{n}$, where $a_{1}, \ldots, a_{n} \in B$, $b_{1}, \ldots, b_{n} \in C$. Similarly, $[B, C]$ is a subset of $P$ consisting of elements of the type $\left[a_{1}, b_{1}\right]+\ldots+\left[a_{n}, b_{n}\right]$, where $a_{1}, \ldots, a_{n} \in B, b_{1}, \ldots, b_{n} \in C$.

Proposition 3. Let $P$ be a Poisson algebra over a field $F$.

(i) If $B$ is a subalgebra of $P$, and if $C$ is an ideal of $P$, then $B+C, B C$ are subalgebras of $P$. Moreover, if $B, C$ are ideals of $P$, then $B+C, B C$ are ideals of $P$.

(ii) $\zeta(P)$ is a subalgebra of $P$, which is an ideal of the Lie algebra $P(+,[]$,$) .$

(iii) $\zeta(P)$ contains every idempotent of $P$, in particular, $1_{P} \in \zeta(P)$.

(iv) If $P$ is not a simple algebra, then $P$ has a proper non-zero maximal ideal.

(v) For every $x \in P$, a subset $x P=\{x y \mid y \in P\}$ is a subalgebra of $P$ and an ideal of $P(+, \cdot)$.

Proposition 4. Let $P$ be a Poisson algebra over a field $F$. Suppose that $A$ is a non-Abelian subalgebra of $P$. If A is nilpotent, then A contains zero divisors.

The Poisson algebra $P$ is called locally nilpotent, if every finitely generated subalgebra of $P$ is nilpotent.

Corollary 1. Let $P$ be a Poisson algebra over a field F. Suppose that $A$ is a locally nilpotent subalgebra of $P$. If A does not contain zero divisors, then A is Abelian. 
Proposition 5. Let $P$ be a Poisson algebra over a field $F$. Suppose that $\operatorname{char}(F)=p$ is a prime, and $F^{p}=F$. If $S$ is a subalgebra of $P$, then the subset $S^{p}=\left\{x^{p} \mid x \in S\right\}$ is a subalgebra of $P$, moreover, $S^{p} \leqslant \zeta(P)$.

Corollary 2. Let $P$ be a Poisson algebra over a field $F$. Suppose that $\operatorname{char}(F)=p$ is a prime, and $F^{p}=F$. Then the subset $P^{p}=\left\{x^{p} \mid x \in P\right\}$ is a subalgebra of $P$, moreover, $P^{p} \leqslant \zeta(P)$.

Corollary 3. Let $P$ be a Poisson algebra over a field $F$. Suppose that $\operatorname{char}(F)=p$ is a prime, and $F^{p}=F$. If $S$ is a finite-dimensional subalgebra of $P$, and if $S$ does not contain zero divisors, then $S \leqslant \zeta(P)$.

Corollary 4. Let $P$ be a Poisson algebra over a field $F$. Suppose that $\operatorname{char}(F)=p$ is a prime, and $F^{p}=F$. If $P$ is finite-dimensional, and if $P$ does not contain zero divisors, then $P$ is Abelian.

We note that every finite field $F$ of characteristic $p$ satisfies $F^{p}=F$. It implies that every locally finite field $F$ of characteristic $p$ satisfies $F^{p}=F$. Thus, we obtain

Corollary 5. Let $P$ be a Poisson algebra over a finite field $F$. If $P$ is finite-dimensional, and if $P$ does not contain zero divisors, then $P$ is Abelian.

Corollary 6. Let $P$ be a Poisson algebra over a locally finite field F. If $P$ is finite-dimensional, and if $P$ does not contain zero divisors, then $P$ is Abelian.

Corollary 7. Let $P$ be a Poisson algebra over a field F. Suppose that $\operatorname{char}(F)=p$ is a prime, and $F^{p}=F$. If $S$ is a locally (finite-dimensional) subalgebra of $P$, and if $S$ does not contain zero divisors, then $S \leqslant \zeta(P)$.

Corollary 8. Let $P$ be a Poisson algebra over a field $F$. Suppose that char $(F)=p$ is a prime, and $F^{p}=F$. If $P$ is locally (finite-dimensional), and if $P$ does not contain zero divisors, then P is Abelian.

Corollary 9. Let $P$ be a Poisson algebra over a finite field F. If P is locally (finite-dimensional), and if $P$ does not contain zero divisors, then $P$ is Abelian.

Corollary 10. Let $P$ be a Poisson algebra over a locally finite field F. If P is locally (finite-dimensional), and if $P$ does not contain zero divisors, then $P$ is Abelian.

The first main result is the following

Theorem 1. Let $P$ be a Poisson algebra over a field F. Suppose that the center of $P$ has a finite codimension $d$. Then $P$ includes an ideal K of a finite dimension of at most $\frac{1}{2} d\left(d^{2}-1\right)$ and such that $P / K$ is Abelian.

Corollary 11. Let $P$ be a Poisson algebra over a field $F$. Suppose that $\operatorname{char}(F)=p$ is a prime, and $F^{p}=F$. If $P$ is finite-dimensional over the center, and if $P$ does not contain zero divisors, then $P$ is Abelian.

Corollary 12. Let $P$ be a Poisson algebra over a finite field F. If P is finite-dimensional over the center, and if $P$ does not contain zero divisors, then $P$ is Abelian.

Corollary 13. Let $P$ be a Poisson algebra over a locally finite field F. If $P$ is finite-dimensional over the center, and if $P$ does not contain zero divisors, then $P$ is Abelian.

In [10], it was proved that if the hypercenter $\zeta_{n}(G)$ of a group $G$ has a finite index, then its $(n+1)$-th hypocenter $\gamma_{n+1}(G)$ is finite. This result was also extended to other algebraic structures, in particular, onto Lie [13] and Leibniz algebras [14, 15]. For the Poisson algebras, we obtain the following

Theorem 2. Let $P$ be a Poisson algebra over a field $F$. Suppose that $\zeta_{n}(P)$ has a finite codimension $d$. Then $P$ includes an ideal K having a finite dimension of at most $d^{n+1}(1+d)$ and such that $P / K$ is nilpotent of a nilpotency class of at most $n$. 
Corollary 14. Let $P$ be a Poisson algebra over a field $F$. Suppose that $\operatorname{char}(F)=p$ is a prime and $F^{p}=F$. If $\zeta_{n}(P)$ has finite codimension, and if $P$ does not contain zero divisors, then $P$ is Abelian.

Corollary 15. Let $P$ be a Poisson algebra over a finite field $F$. If $\zeta_{n}(P)$ has finite codimension, and if $P$ does not contain zero divisors, then $P$ is Abelian.

Corollary 16. Let $P$ be a Poisson algebra over a locally finite field $F$. If $\zeta_{n}(P)$ has finite codimension, and if $P$ does not contain zero divisors, then $P$ is Abelian.

Now, we present our last result.

Theorem 3. Let $P$ be a finitely generated Poisson algebra over a field $F$, and let $K$ be an ideal of $P$. If $K$ has a finite codimension, then $K$ is finitely generated as an ideal.

\section{REFERENCES}

1. Lichnerowicz, A. (1977). Les variétés de Poisson et leurs algèbres de Lie associées. J. Differential Geom., 12, No. 2, pp. 253-300. https://doi.org/10.4310/jdg/1214433987

2. Weinstein, A. (1977). Lecture on symplectic manifolds. CBMS regional conference series in mathematics, No. 29. Providence, R.I: Amer. Math. Soc. https://doi.org/10.1090/cbms/029

3. Berezin, F. A. (1967). Some remarks about the associated envelope of a Lie algebra. Funct. Anal. Appl., 1, No. 2, pp. 91-102. https://doi.org/10.1007/BF01076082

4. Vergne, M. (1972). La structure de Poisson sur l'algèbre symétrique d'une algèbre de Lie nilpotente. Bull. Soc. Math. France, 100, pp. 301-335.

5. Braconnier, J. (1977). Algèbres de Poisson, C.R. Acad. Sci., A, 284, No. 21, pp. 1345-1348.

6. Prosser, R. T. (1977). Poisson brackets and commutator brackets. I. Proc. Amer. Math. Soc., 62, No. 2, pp. 305-309. https://doi.org/10.1090/S0002-9939-1977-0443739-1

7. Prosser, R. T. (1977). Poisson brackets and commutator brackets. II. Proc. Amer. Math. Soc., 62, No. 2, pp. 310-315. https://doi.org/10.1090/S0002-9939-1977-0443740-8

8. Atkin, C. J. (1984). A note on the algebra of Poisson brackets. Math. Proc. Cambridge Philos. Soc., 96, No. 1, pp. 45-60. https://doi.org/10.1017/S0305004100061922

9. Neumann, B. H. (1951). Groups with finite classes of conjugate elements. Proc. Lond. Math. Soc., 3, No. 1, pp. 178-187. https://doi.org/10.1112/plms/s3-1.1.178

10. Baer, R. (1952). Endlichkeitskriterien für Kommutatorgruppen. Math. Ann., 124, No. 1., pp. 161-177. https://doi.org/10.1007/BF01343558.

11. Kurdachenko, L. A. \& Subbotin, I. Ya. (2016). On the relationships between the factors of upper and lower central series in groups and other algebraic structures. Note Mat., 36, No. 1, pp. 35-50. https://doi.org/10.1285/ i15900932v36suppl1p35

12. Vaughan-Lee, M. R. (1972). Metabelian BFC p-groups. J. Lond. Math. Soc., 5, No. 4, pp. 673-680. https:// doi.org/10.1112/jlms/s2-5.4.673

13. Kurdachenko, L. A., Pypka, A. A. \& Subbotin, I. Ya. (2015). On some relations between the factors of the upper and lower central series in Lie algebras. Serdica Math. J., 60, No. 2-3, pp. 293-306.

14. Kurdachenko, L. A., Otal, J. \& Pypka, A. A. (2016). Relationships between factors of canonical central series of Leibniz algebras. Eur. J. Math., 2, No. 2, pp. 565-577. https://doi.org/10.1007/ s40879-016-0093-5

15. Kurdachenko, L. A., Otal, J. \& Subbotin, I. Ya. (2019). On some properties of the upper central series in Leibniz algebras. Comment. Math. Univ. Carolin., 60, No. 2, pp. 161-175. https://doi.org/10.14712/12137243.2019 .009

Received 20.02.2021 
Л.А. Курдаченко ${ }^{1}$, https://orcid.org/0000-0002-6368-7319

O.O. Пипка ${ }^{1}$, https://orcid.org/0000-0003-0837-5395

I.Я. Субботін ${ }^{2}$, https://orcid.org/0000-0002-6242-3995

${ }_{1}^{1}$ Дніпровський національний університет ім. Олеся Гончара

${ }^{2}$ Національний університет, Лос-Анджелес, США

E-mail: lkurdachenko@gmail.com, sasha.pypka@gmail.com, isubboti@nu.edu

ПРО АНАЛОГИ ДЕЯКИХ КЛАСИЧНИХ

ТЕОРЕТИКО-ГРУПОВИХ РЕЗУЛЬТАТІВ

В АЛГЕБРАХ ПУАССОНА

Досліджено алгебри Пуассона, в яких $n$-й гіперцентр (центр) має скінченну ковимірність. Встановлено, що в цьому випадку алгебра Пуассона $P$ містить такий скінченновимірний ідеал $K$, що $P / K$ нільпотентна (абелева). Більше того, якщо $n$-й гіперцентр алгебри Пуассона $P$ над деяким полем має скінченну ковимірність і $P$ не містить дільників нуля, то $P$ абелева.

Ключові слова: алгебра Пуассона, центр, гіперцентр, дільник нуля, нільпотентність. 